\title{
Modeling methodology for an HTS flux pump using 2D
}

\author{
$\boldsymbol{H}$-formulation \\ Jianzhao Geng ${ }^{\text {a) }}$, and T. A. Coombs \\ Department of Engineering, University of Cambridge, Cambridge CB3 OFA, United Kingdom
}

a) Email: jg717@cam.ac.uk

\begin{abstract}
Flux pumps are the kind of devices that can magnetize closed superconducting magnets in a gradual manner. High- $T_{\mathrm{c}}$ Superconducting (HTS) flux pumps are particularly promising for high field applications, due to the fact that lossless HTS coils are unavailable. The physics of these devices is also attractive. In this paper, we propose a modeling methodology for a transformer-rectifier HTS flux pump switched by dynamic resistance. A finite element model is built in Comsol and solved by 2D $H$-formulation. The simulation result is verified by experimental data. The simulation will give a clear picture of how flux pumping occurs in the superconductor. It will show flux motion across a superconductor by shifting the electric central line, which is a unique nature of type-II superconductors. This work may be interesting in the understanding of magnetization of High- $T_{\mathrm{c}}$ Superconductors.
\end{abstract}

\section{Introduction}

Flux pumps are the kind of devices that can magnetize closed superconducting magnets without electrical contact. They are ideal alternatives to the bulky electronic power sources in powering high-current high-field superconducting magnets. It is also tantalizing to understand the physics behind these devices where the concept of flux motion, resistance, and electric field in the diamagnetic material is in line with Faraday's Law.[1]

Low- $T_{\mathrm{c}}$ Superconducting (LTS) flux pumps [2][3] have been developed for decades. In these devices the superconductor is at least partially driven normal to allow flux motion. In HTS flux pumps [4-8], however, it is not practical to drive the superconductor normal by magnetic fields, due to the very high irreversibility field [9] of high-temperature superconductors. In our previous work [10], we explained two ways for HTS flux pump to work. The first [8] is to exceed the local critical current, either by inducting a current higher than the critical value, or by reducing the critical current density using external field. The second [7] is to take advantage of dynamic resistance effect [11-18]. When a type II superconductor carrying a direct current is subjected to a perpendicular oscillating magnetic field, vortex in the superconductor will redistribute and flux will travel across the superconductor. This effect occurs when the superconductor is fully penetrated by the transport current and the oscillating magnetic field. It should be noted that this effect is very different from the flux flow caused by de-pinning [19]. It is not necessary to exceed the local critical current density for flux to move. Therefore, this effect not only exists in superconductors with nonlinear E-J curve (e.g. E-J power law [20]), but also exists under the Critical State Model (CSM) [21], where the current density inside a superconductor can only be \pm $J_{\mathrm{c}}$ or 0 . 
Recently, Campbell [22] proposed a finite element model to calculate flux pumping using 2D A-formulation. His result showed that flux pumping occurs under CSM, and field dependence of $J_{\mathrm{c}}$ facilitates flux pumping. His result, however, did not show that flux pumping is associated with the dynamic resistance effect. In Ref. [23] and Ref. [24], modeling technique for dynamic resistance was proposed using $T$-formulation and $H$-formulation respectively. In this paper, we further these studies to show how dynamic resistance incurred flux pumping can be simulated using $H$-Formulation with finite element software. We will present a clear picture of how flux travel across a superconductor transporting no direct current.

\section{Modeling methodology}

\subsection{Geometry description}

(a)

Applied field area

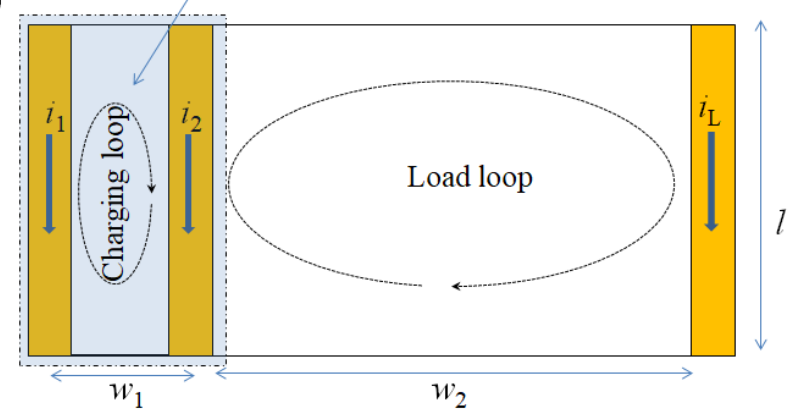

(b)

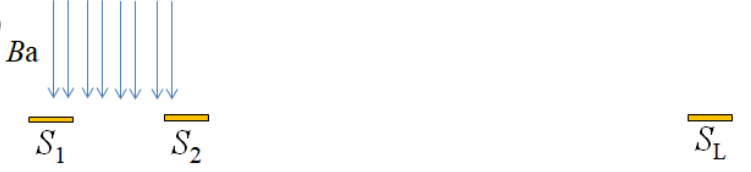

Fig. 1. Schematic drawing of the HTS flux pumping circuit to be simulated. (a) Three thin superconducting strips $\left(S_{1}, S_{2}\right.$, and $\left.S_{\mathrm{L}}\right)$ are set in parallel with their terminations connected together. Two loops, a smaller charging loop and a larger load loop are formed. The field is applied perpendicular to the charging loop area. Flux pumping is considered to occur if the load loop current $\left(i_{\mathrm{L}}\right)$ is gradually charged up. (b) cross-section view.

The circuit for simulation consists of three parallel HTS strips, with their terminations connected together, as shown in Fig. 1(a). The three strips are denoted by $S_{1}, S_{2}$ and $S_{\mathrm{L}}$ respectively. The currents in these three strips are denoted by $i_{1}, i_{2}$, and $i_{\mathrm{L}}$ respectively, with the reference direction in Fig. 1(a). The closed loop formed by $S_{1}$ and $S_{2}$ is called the charging loop, which has a width of $w_{1}$. The third strip $S_{\mathrm{L}}$ is set far apart from $S_{1}$ and $S_{2}$. The loop formed by $S_{\mathrm{L}}$ and the other two strips is defined as the load loop, which has a width of $w_{2}$. The length of the three strips is denoted by $l$. We assume that the load loop is much larger than the charging loop, $\mathrm{i}$. e. $w_{2}>>w_{1}$, and the length of the strips is much larger than the width of loops, i. e. $l>>w_{2}$. Assuming that the magnetic field is applied only to the area of the charging loop, normal to the page, with uniform field strength along $l$. Based on these assumptions, we may neglect the terminations of strips, and can take an arbitrary cross section (normal to strip length) for study, as shown in Fig. 1(b). Then the problem is converted to a 2 dimensional issue. The flux pumping effect is considered to happen if magnetic field is only applied at the small charging loop area, and the load loop can be magnetized ( $i_{\mathrm{L}}$ gradually increases).

We built a 2D infinitely long (into the page) model in Comsol PDE, the geometry is shown in 
Fig. 2.

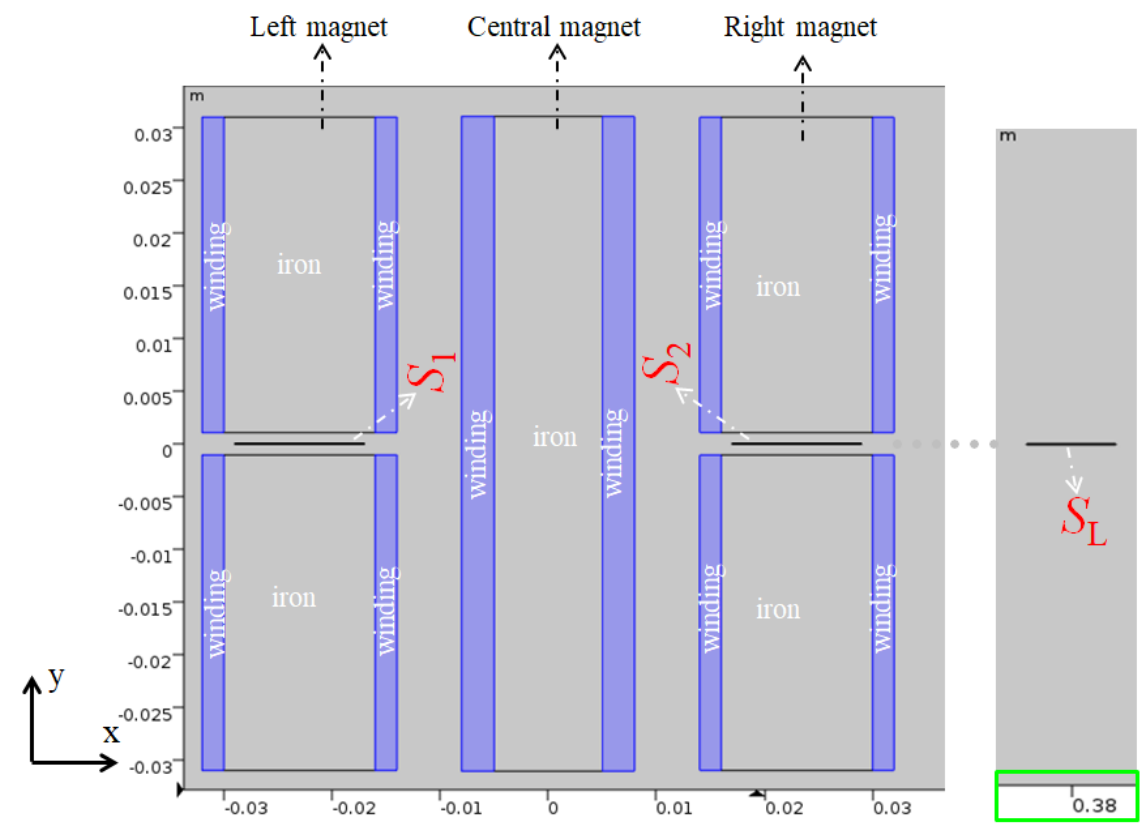

Fig. 2. The 2D infinitely long model simulating flux pumping effect in Comsol. $S_{1}, S_{2}$ and $S_{\mathrm{L}}$ indicate the three HTS strips. The central electromagnet goes through the gap between $S_{1}$ and $S_{2}$, which is used to generate a circulating screening current in between $S_{1}$ and $S_{2}$. The left magnet and right magnet are used to generate magnetic fields perpendicular to $S_{1}$ and $S_{2}$, referred as switching fields. It should be noted that the strip $S_{\mathrm{L}}$ is actually far away from the other two strips in the model, and in order to let readers view the geometry clearly they are shown close to each other.

The width of HTS strip $S_{1}$ and $S_{2}$ is $12 \mathrm{~mm}$, and the width of $S_{\mathrm{L}}$ is $8 \mathrm{~mm} . S_{1}$ and $S_{2}$ are $46 \mathrm{~mm}$ apart from each other, whereas $S_{\mathrm{L}}$ is about $360 \mathrm{~mm}$ away from $S_{2}$. It should be noted that in Fig. 2 the distance between $S_{2}$ and $S_{\mathrm{L}}$ is shortened for the convenience of drawing.

Only the superconducting layer is simulated, and other layers (substrate, stabilizer, buffer layers) are neglected. The thickness of each strip is chosen to be $100 \mu \mathrm{m}$, which is 100 times of the real thickness. The thickness enlargement will sacrifice accuracy but will not change the fundamentals.

Three electromagnets are used to apply fields to the charging loop. Each electromagnet consists of a pair of windings and an iron core. The central magnet goes through the gap between $S_{1}$ and $S_{2}$, which acts as a transformer inducing circulating current around $S_{1}$ and $S_{2}$. Each of the left and right magnets consists of a top pole and a bottom pole, with a $1 \mathrm{~mm}$ gap to fit in $S_{1}$ and $S_{2}$. Thus they can apply magnetic fields normal to the surface of $S_{1}$ and $S_{2}$, acting as switches. The magnetic fields generated by the left and right magnets are denoted by switching fields. The whole area for simulation is a $2 \mathrm{~m} \times 2 \mathrm{~m}$ square, and the rest part is considered to be free space.

A mapped mesh is used in the superconducting strips, with 50 elements uniformly distributed along the width of each strip, and 5 elements uniformly distributed along the thickness of each strip. In the rest of the area a free triangular mesh is used.

\subsection{D H-formulation}

For the proposed geometry, the 2-D $H$-formulation [25-28] is used for the simulation. In the 
$H$-formulation, the magnetic field $\boldsymbol{H}$ is used to solve the Maxwell Equation, i. e. the Faraday's Law (1) and the Ampere's Law (2). By substituting the Ohm's Law (3) and magnetic property (4) into Eq. 1 and Eq. 2, one can eliminate all variables except $\boldsymbol{H}$, and acquire Eq. 5.

$$
\begin{gathered}
\nabla \times \boldsymbol{E}=-\frac{\partial \boldsymbol{B}}{\partial t}(1) \\
\nabla \times \boldsymbol{H}=\boldsymbol{J} \quad(2) \\
\boldsymbol{E}=\rho \boldsymbol{J} \quad(3) \\
\boldsymbol{B}=\mu_{0} \mu_{\mathrm{r}} \boldsymbol{H} \quad(4) \\
\frac{\partial\left(\mu_{0} \mu_{\mathrm{r}} \boldsymbol{H}\right)}{\partial t}+\nabla \times(\nabla \times \rho \boldsymbol{J})=0
\end{gathered}
$$

where $\boldsymbol{E}=\left[E_{z}\right]$ represents the electric field, $\boldsymbol{B}=\left[B_{x}, B_{y}\right]$ is the flux density, $\boldsymbol{H}=\left[H_{x}, H_{y}\right]$ is the magnetic field strength, and $\boldsymbol{J}=\left[J_{\mathrm{Z}}\right]$ is the current density. $\rho$ is resistivity, $\mu_{0}$ is the permeability of free space, and $\mu_{\mathrm{r}}$ is the relative permeability.

To solve Eq. 5, the relative permeability and resistivity are needed for each material. For the sub-domain of iron cores, the relative permeability is set to be $\mu_{\mathrm{r}}=1000$, and for all other subdomains $\mu_{\mathrm{r}}=1$. The resistivity of air domain is set to be $\rho=1 \Omega \mathrm{m}$, for iron subdomains it is set to be $\rho=0.01 \Omega \mathrm{m}$, and for the winding subdomains it is set to be $\rho=10^{-8} \Omega \mathrm{m}$. The resistivity of superconductors follows the E-J power law [20]:

$$
\boldsymbol{E}=E_{0}\left(\frac{\boldsymbol{J}}{J_{c}}\right)^{n}
$$

Where $E_{0}$ is $10^{-4} \mathrm{~V} / \mathrm{m}$, and $n=21$. The value of $J_{\mathrm{c}}$ is set to be $2.5 \times 10^{2} \mathrm{~A} / \mathrm{mm}^{2}$, and the critical current is $300 \mathrm{~A}$ in strips $S_{1}$ and $S_{2}$, and is $200 \mathrm{~A}$ in strip $S_{\mathrm{L}}$. Here we intentionally do not consider any field dependence of critical current density. The aim to do so is to show that pure dynamic resistance is sufficient to result in flux pumping. We want to distinguish this effect from the field dependence of $J_{\mathrm{c}}$ induced flux pumping effect, which has been predicted [10] and experimentally verified [8] in our previous work, and numerically verified by Campbell [22]. The boundary condition for the superconductors is

$$
\boldsymbol{i}_{1}+\boldsymbol{i}_{2}+\boldsymbol{i}_{L}=\int_{S 1} \boldsymbol{J} d S+\int_{S 2} \boldsymbol{J} d S+\int_{S L} \boldsymbol{J} d S=0
$$

Eq. 7 indicates that although the current in each of the three superconducting strips is unknown, by forcing the sum of the three currents to be zero, the three superconductors are forming a circuit which looks like that in Fig. 1(a). The boundary condition is achieved by using point-wise constraint in the superconducting domains.

The outer boundaries which are far from the area of interest is set to be Zero Flux.

\subsection{Generation of magnetic fields}

Magnetic field is applied by setting the current in the windings, and each pair of windings wound around the same iron core have the same current magnitude but opposite in direction (current flows in from one winding and flows out from the other). The top windings and bottom windings of the left (or right) magnet have the same current, so that in each air gap the magnetic 
field is mainly normal to the superconducting strip. The current is applied by integrating the current density in each winding area, and constraining it to a certain value. Specifically, the left magnet current $i_{\text {left, }}$, the central magnet current $i_{\text {cent }}$ and the right magnet current $i_{\text {right }}$ are set to be:

$$
\left\{\begin{array}{c}
\boldsymbol{i}_{\text {left }}=A_{1} \times \sin (40 \pi t), \text { for } 0.725<\bmod (t, 1)<0.775 \text { and } t>1 \\
\boldsymbol{i}_{\text {cent }}=A_{2} \times \sin (2 \pi t) \\
\boldsymbol{i}_{\text {right }}=A_{1} \times \sin (40 \pi t), \text { for } 0.225<\bmod (t, 1)<0.275 \text { and } t>1
\end{array}\right.
$$

Where $A_{1}$, and $A_{2}$ represent the magnitudes.
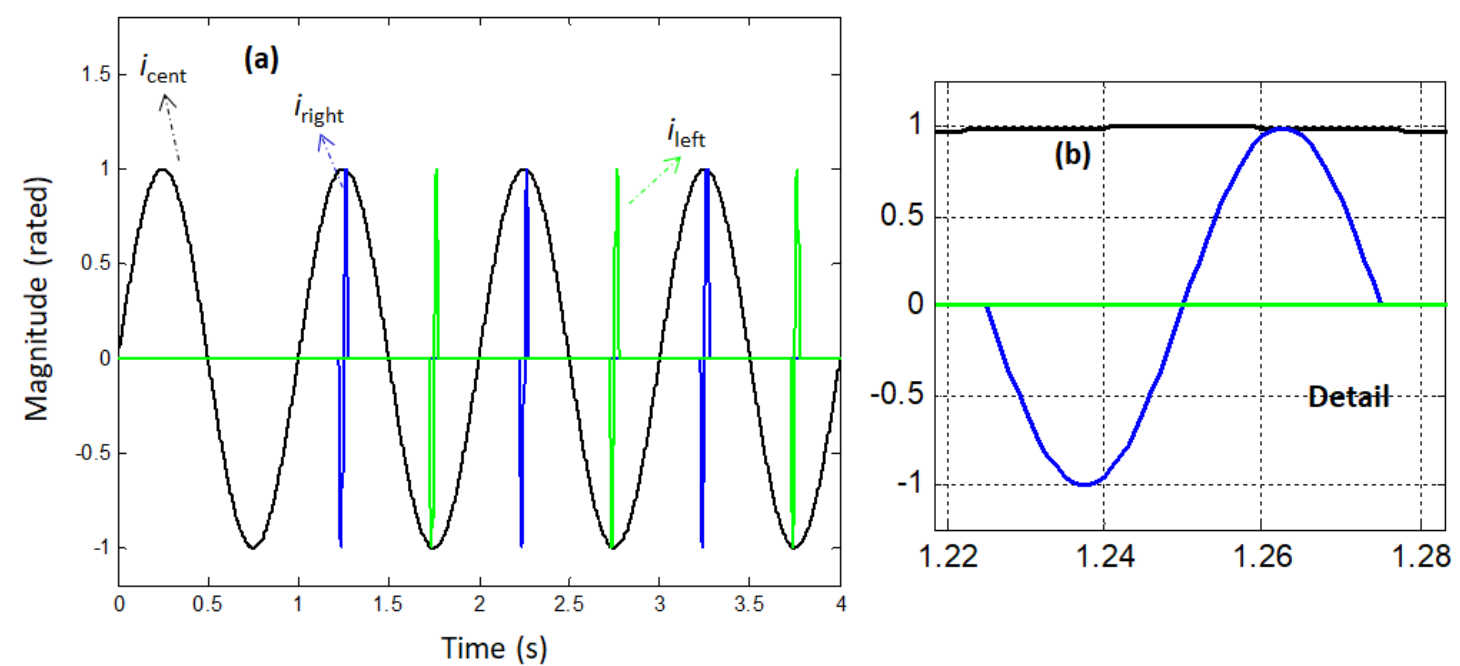

Fig. 3. Waveform of the three currents driving the magnets. The central magnet is driven by a $1 \mathrm{~Hz}$ current, the right magnet is driven by an intermittent $20 \mathrm{~Hz}$ current which is only applied one cycle at each positive peak of the central-magnet current, and the left magnet is driven by an intermittent $20 \mathrm{~Hz}$ current which is only applied one cycle at each negative peak of the central-magnet current. (a) overall waveform, (b) detail of one ac switching field cycle. (the current magnitudes do not reflect the actual value)

The waveform of these currents is plotted in Fig. 3. The central magnet current is a $1 \mathrm{~Hz}$ pure sine wave. The current in the left magnet and right magnet is zero, except for a single cycle of a 20 $\mathrm{Hz}$ sine wave which occurs at each positive and negative peak of the central magnet current. During the first second, only the central-magnet current is applied. This is to verify that symmetrical current in the central magnet will not result in flux pumping if there are no switching field applied. In the following simulation, $A_{2}$ in Eq. 8 is adjusted to $900 \mathrm{~A}$ so that the induced screening current in $S_{1}$ and $S_{2}$ is with around 200A peak value, and the switching-magnet current is changeable.

It should be noted that the actually applied magnetic fields slightly lag the driving currents, because the current distribution transience in the windings.

The model has been running on a desktop with a $3.5 \mathrm{GHz}$ CPU and a 32 GB memory. In each run, 9 seconds was simulated. Each run takes about 70 to 120 hours depending on different applied field magnitudes.

\section{3 simulation result and analysis}




\subsection{Charging curves}

Fig. 4(a) shows the current curves in the three superconducting strips, with the switching-magnet current magnitude $A_{1}$ in Eq. 8 to be $500 \mathrm{~A}$. We can see that during the first second when only the central magnet is activated there is a screening current flowing around $S_{1}$ and $S_{2}$, the current $i_{\mathrm{L}}$ in $S_{\mathrm{L}}$ is very small. After that, the switching magnetic fields are alternatively applied to either $S_{1}$ or $S_{2}$ when the screening current in the strip is approaching its positive peak. Each time the switching field is applied, there is a net increase in the load current $i_{\mathrm{L}}$, although there is an oscillation. At the same time, the current in the switched strip drops sharply. With the load current $i_{\mathrm{L}}$ ramping up, each step of increase in $i_{\mathrm{L}}$ becomes smaller. The load current saturates at about 180 A which is close to the peak value of the screening current in strips $S_{1}$ and $S_{2}$ before applying the switching field. The $i_{\mathrm{L}}$ curve looks like the charging curve of a first order circuit. Fig. 4(b) shows the experimental charging curve of an AC field switched transformer-rectifier HTS flux pump [7][29] for comparison. The experiment is different from the simulation in the following aspect: it was a half-bridge rectifier rather than a full-bridge one, only one switch was used rather than two, the transformer current is a triangular wave rather than a sine wave, the transformer and the switching magnet have limited length, and the load was a 40 turn coil rather than a single strip. Despite these differences, the simulation and experimental charging curves are similar except for the charging speed. In the experiment the load coil inductance is much higher than the inductance of the strip, so the charging time is significantly longer.

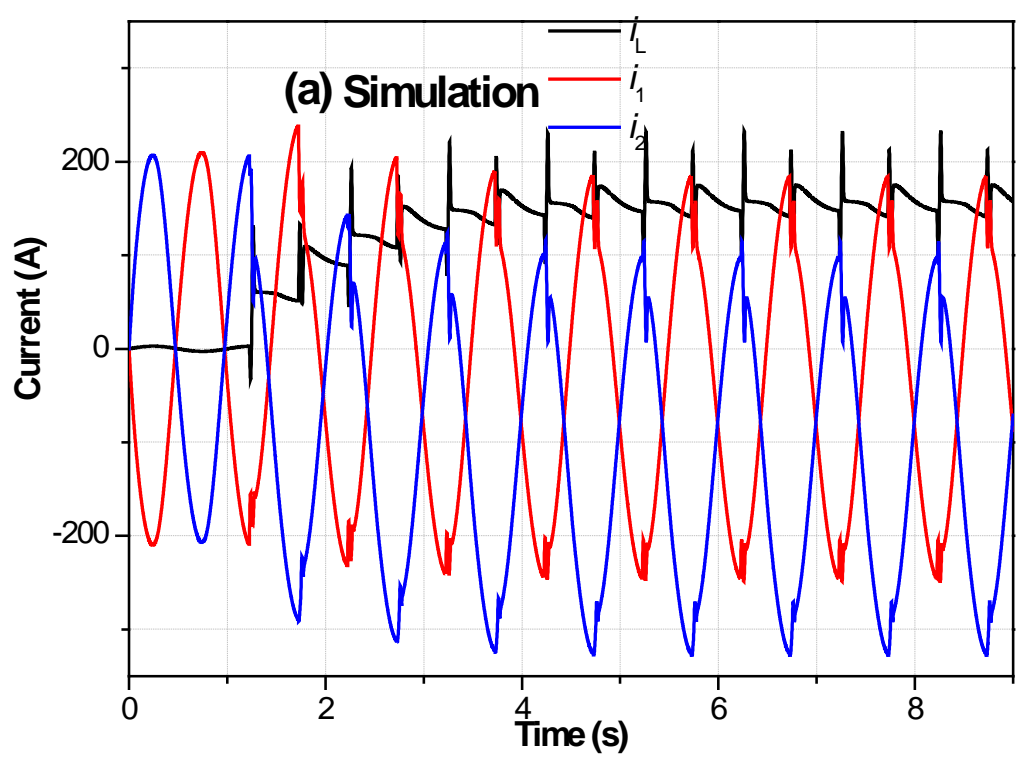




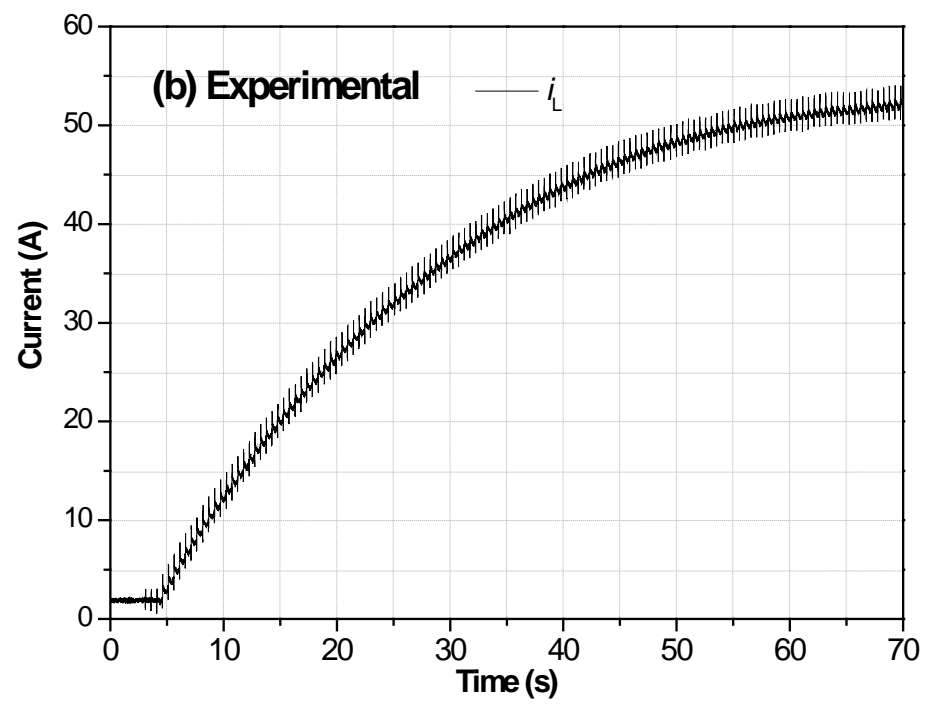

Fig. 4. Simulation and experimental results show flux pumping. (a) simulation result showing the waveform of $i_{1}$, $i_{2}$, and $i_{\mathrm{L}}$. (b) experimental result showing the charging curve of a transformer-rectifier flux pump switched by dynamic resistance .
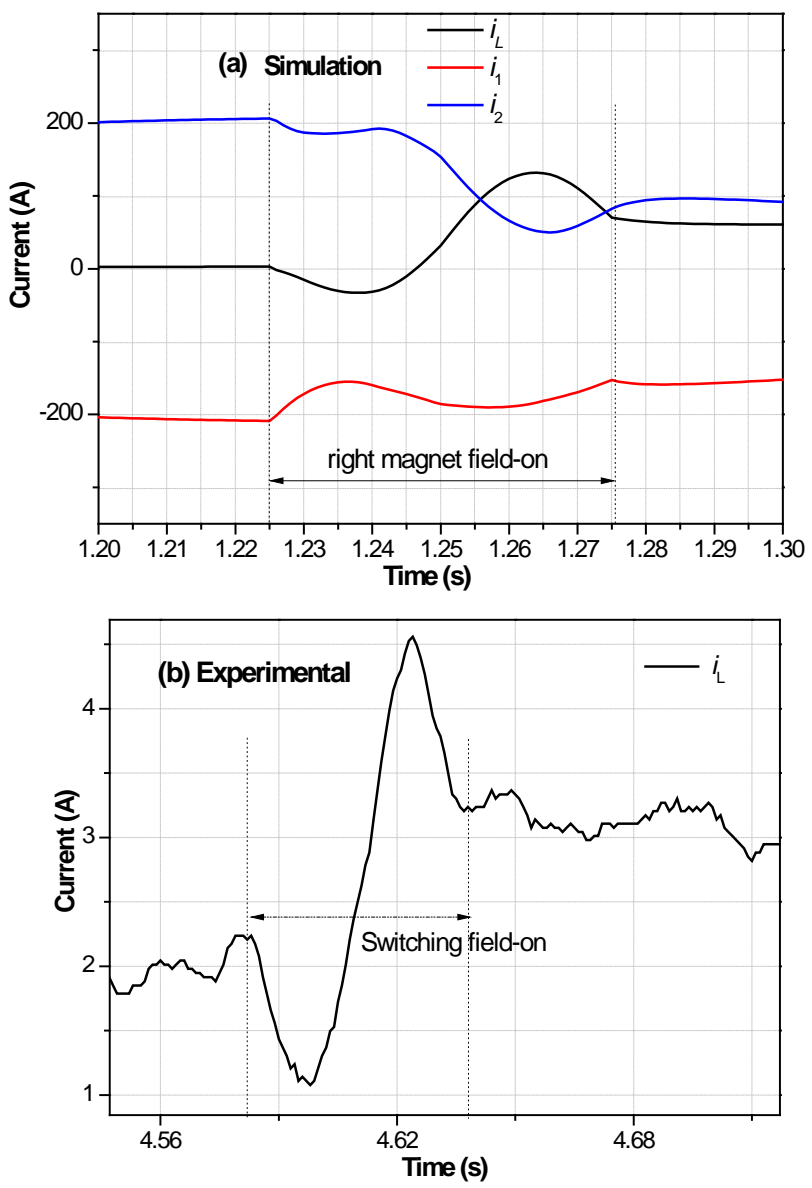

Fig. 5. Simulation and experimental result comparison during a single period of the switching field. (a) simulation result, (b) experimental result.

To investigate the details, we enlarged Fig. 4 to display the time of a switching field cycle, as shown in Fig. 5. The figure clearly shows that the simulation waveform is in accordance with the 
experimental data. After applying the switching field, there is an increase in the load current $i_{\mathrm{L}}$.

We also investigated the influence of switching fields magnitudes on the flux pumping performance. In the simulation we chose the value of $A_{1}$ in Eq. 8 to be $100 \mathrm{~A}, 200 \mathrm{~A}$, and 500A respectively. As shown in Fig. 6, higher field magnitude results in faster charging as well as a higher final saturation load current. When the switching field is too low, a very little load current is pumped, this is because the switching field can barely overcome the threshold field. This will be discussed in next section.

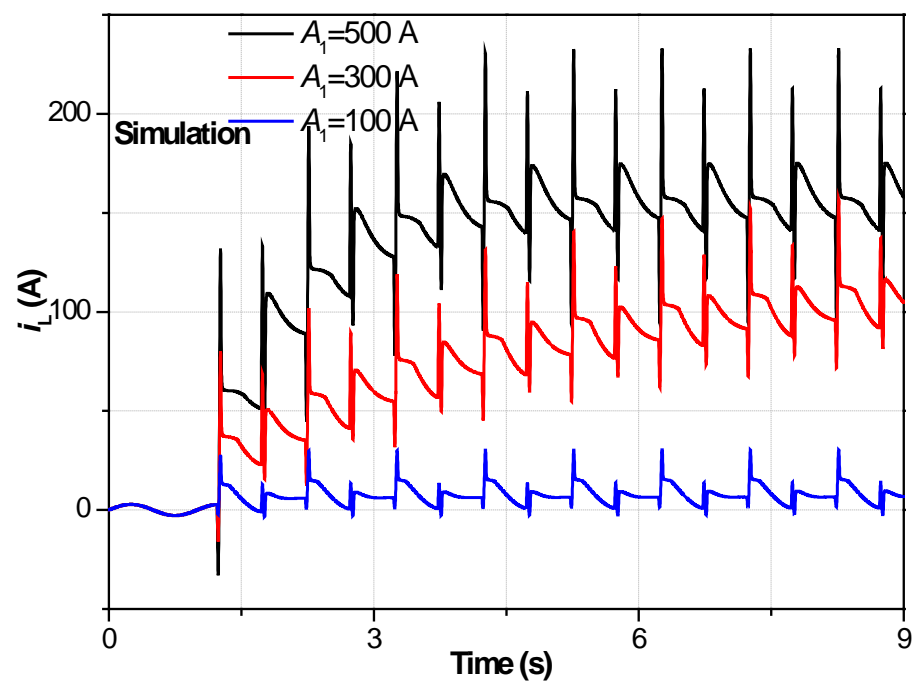

Fig. 6. Simulation result of $i_{\mathrm{L}}$ ramping under different magnitudes of switching fields.

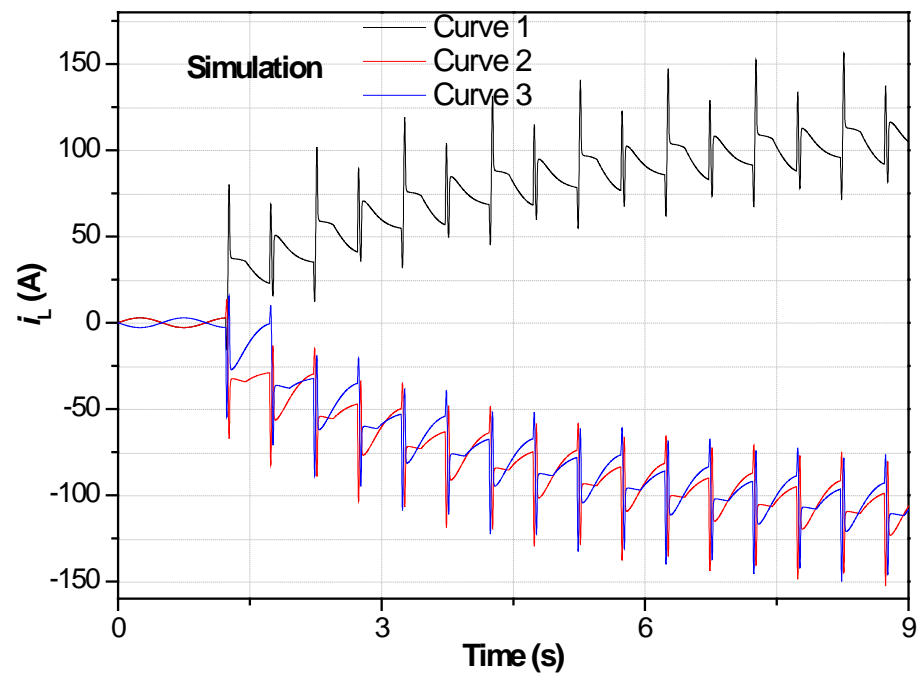

Fig. 7. Simulation result of $i_{\mathrm{L}}$ ramping under different current sequences. In Curve 1, the switching sequence is the same with that described in Eq. 8; in Curve 2, the switching sequence of $i_{\text {left }}$ and $i_{\text {right }}$ exchanges whereas $i_{\text {cent }}$ keeps unchanged, so that $i_{\text {left }}$ and $i_{\text {right }}$ are applied around the negative peaks of $i_{\text {cent }}$; in Curve 3, switching sequence of $i_{\text {left }}$ and $i_{\text {right }}$ keeps unchanged, but the phase of $i_{\text {cent }}$ changes 180 degree so that $i_{\text {left }}$ and $i_{\text {right }}$ are also applied around the negative peaks of $i_{\text {cent }}$. In all three occasions the magnitude of $i_{\text {left }}$ and $i_{\text {right }}$ is $300 \mathrm{~A}$, and the magnitude of $i_{\text {cent }}$ is 900 A.

Another set of simulation is done concerning the flux pumping polarity which is considered important. The load current curves are shown in Fig. 7 under three different field applying sequences. In Curve 1, the applying field sequence is the same as described in Fig. 3. In Curve 2, 
the current $i_{\text {cent }}$ is the same as described in Fig. 3 , but $i_{\text {left }}$ and $i_{\text {right }}$ exchange order. In this case, both $i_{\text {left }}$ and $i_{\text {right }}$ are applied around the negative peaks of $i_{\text {cent }}$. In Curve 3, the currents $i_{\text {left }}$ and $i_{\text {right }}$ are the same as described in Fig. 3, whereas the phase of $i_{\text {cent }}$ changes $180^{\circ}$, so that the currents $i_{\text {left }}$ and $i_{\text {right }}$ are also applied around the negative peaks of $i_{\text {cent }}$. The load current in Curve 2 and Curve 3 become negative, indicating the flux motion is only dependent on the direction of screening current around strip $S_{1}$ and $S_{2}$ when the switching field is applied. This is in accordance with the classic dynamic resistance model. The minor difference between Curve 2 and Curve 3 is due to the fact that the geometry is not strictly symmetrical. The right magnet is closer to the strip $S_{\mathrm{L}}$ than the left magnet, so the inductions of these two magnets are different.

\subsection{Current and magnetic field distribution}

To further understand the electro-magnetic behavior in the superconductors, we investigated the current and magnetic field distribution in the strips. In all the following results, the thickness of each strip is intentionally enlarged for a clearer view.

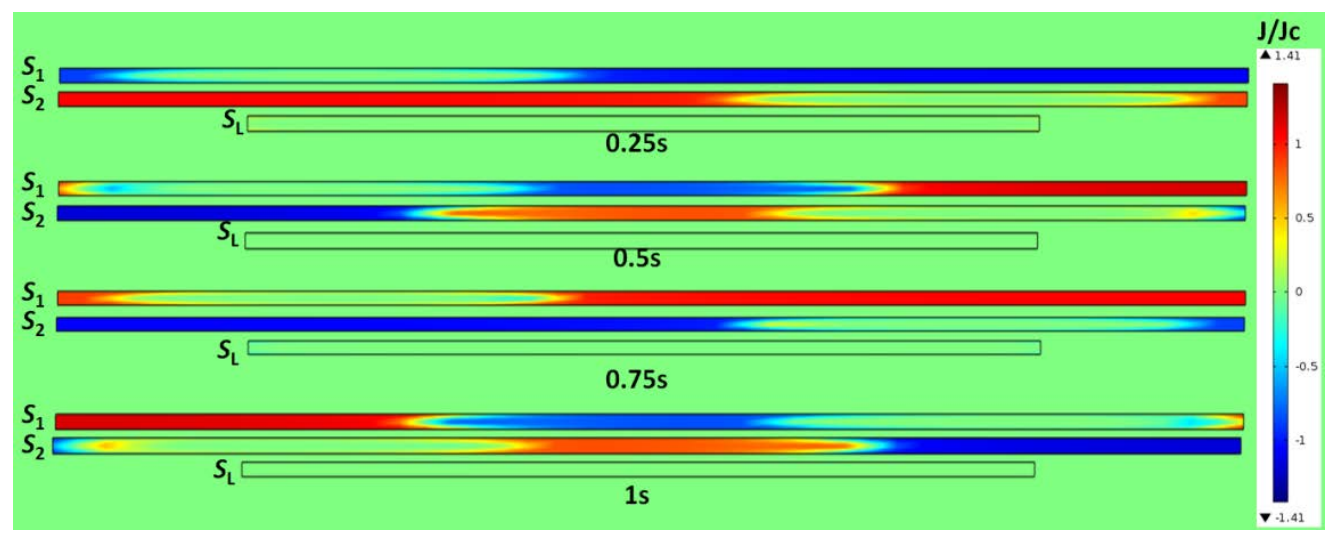

Fig. 8. Current distribution in the three superconducting strips during the first cycle of the central-magnet current changes. The time is corresponding to the curve in Fig. 3. The thickness of each strip is intentionally enlarged to present a clearer view.

Fig. 8 shows the current distribution in the three superconducting strips during one cycle of the central-magnet current, during which the left and right magnets are not activated. For the strips $S_{1}$ and $S_{2}$ which enclose the central magnet, most of the current tend to circulate the charging loop in the inner part, and only a little portion of the current flows near the edge. There is very little current flowing through the strip $S_{\mathrm{L}}$ because on one hand it is far away from the central magnet, and on the other hand the induction by the central magnet is shielded by the strip $S_{1}$ and $S_{2}$.

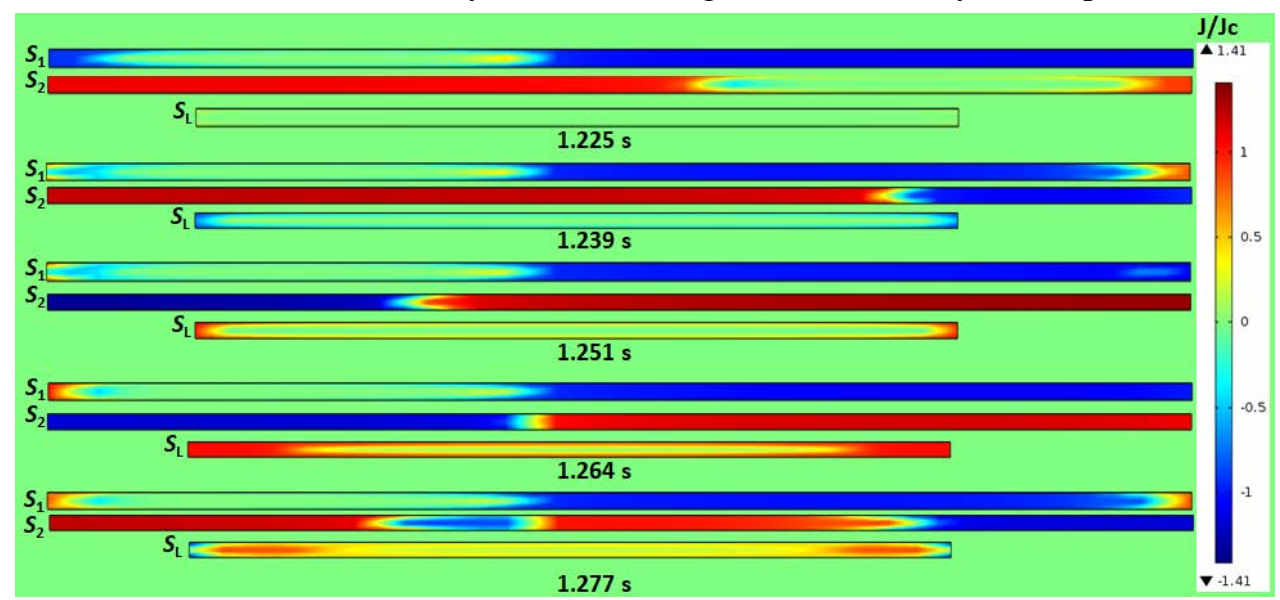


Fig. 9. Current distribution in the three superconducting strips during the first cycle of applied field on $S_{2}$ by the right magnet.

Fig. 9 shows the current distribution in the three superconducting strips during the first cycle of applied field on the strip $S_{2}$. It is noted that the frequency of $i_{\text {right }}$ is $20 \mathrm{~Hz}$, so its duration is $0.05 \mathrm{~s}$. However, there is a slight delay in the applied field compared to the current, because transitional current redistribution in the windings. The current is applied from $1.225 \mathrm{~s}$ to $1.275 \mathrm{~s}$, whereas as the field response is from $1.225 \mathrm{~s}$ to $1.277 \mathrm{~s}$. At the time point $1.225 \mathrm{~s}$, the field has not been applied to the strip, so little current is flowing through $S_{\mathrm{L}}$, and the currents in $S_{1}$ and $S_{2}$ look symmetrical. After this time point the switching field of $S_{2}$ starts to decrease to its negative peak at $1.239 \mathrm{~s}$. During this period, screening current induced by the switching magnet penetrates the strip $S_{2}$ from both edges. Because the central magnet has already induced a current (can be approximately considered as a "transport current") in the strip, the current distribution in the strip is not symmetrical. As can be seen from the 1.239 s scene in Fig. 9, the electric central line (between opposite side of which the electric field changes directions) in $S_{2}$ is biased to the right. Another detail worth paying attention is that due to the flux penetration into the strip $S_{2}$, flux linking the strip $S_{1}$ and $S_{\mathrm{L}}$ changes, resulting in a corresponding current change. The current in the strip $S_{1}$ changes color at both edges, indicating a current decrease; whereas a blue current occurs at the outer part of the strip $S_{\mathrm{L}}$, also indicating a flux change. After 1.239s, the applied field starts to increase, the induced screening current changes direction. However, because of the existence of the "transport current", the electric central line in the strip has been shifted to the left. It is clearly seen that at $1.25 \mathrm{~s}$ the current in $S_{\mathrm{L}}$ has changed direction, and at $1.264 \mathrm{~s}$ it increased to a noticeable value. The point $1.264 \mathrm{~s}$ is when the applied field reaches its positive peak, and after that it decreases to zero at $1.277 \mathrm{~s}$, completing an entire cycle. It can be seen that although the current in $S_{\mathrm{L}}$ has a slight decrease from $1.264 \mathrm{~s}$ to $1.277 \mathrm{~s}$, its final value is still much larger than its initial value at $1.225 \mathrm{~s}$, indicating flux pumping has been achieve. Similar result is observed when the field is applied to the strip $S_{1}$ too.

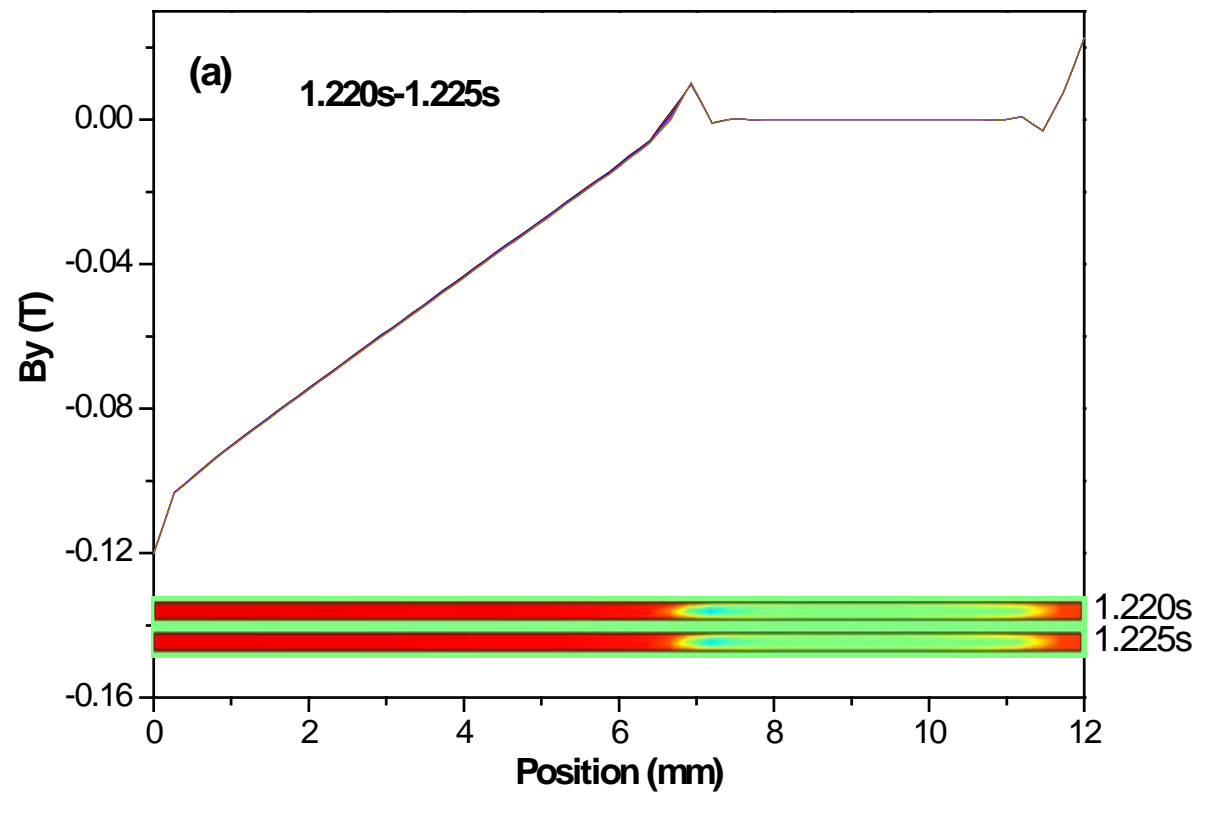



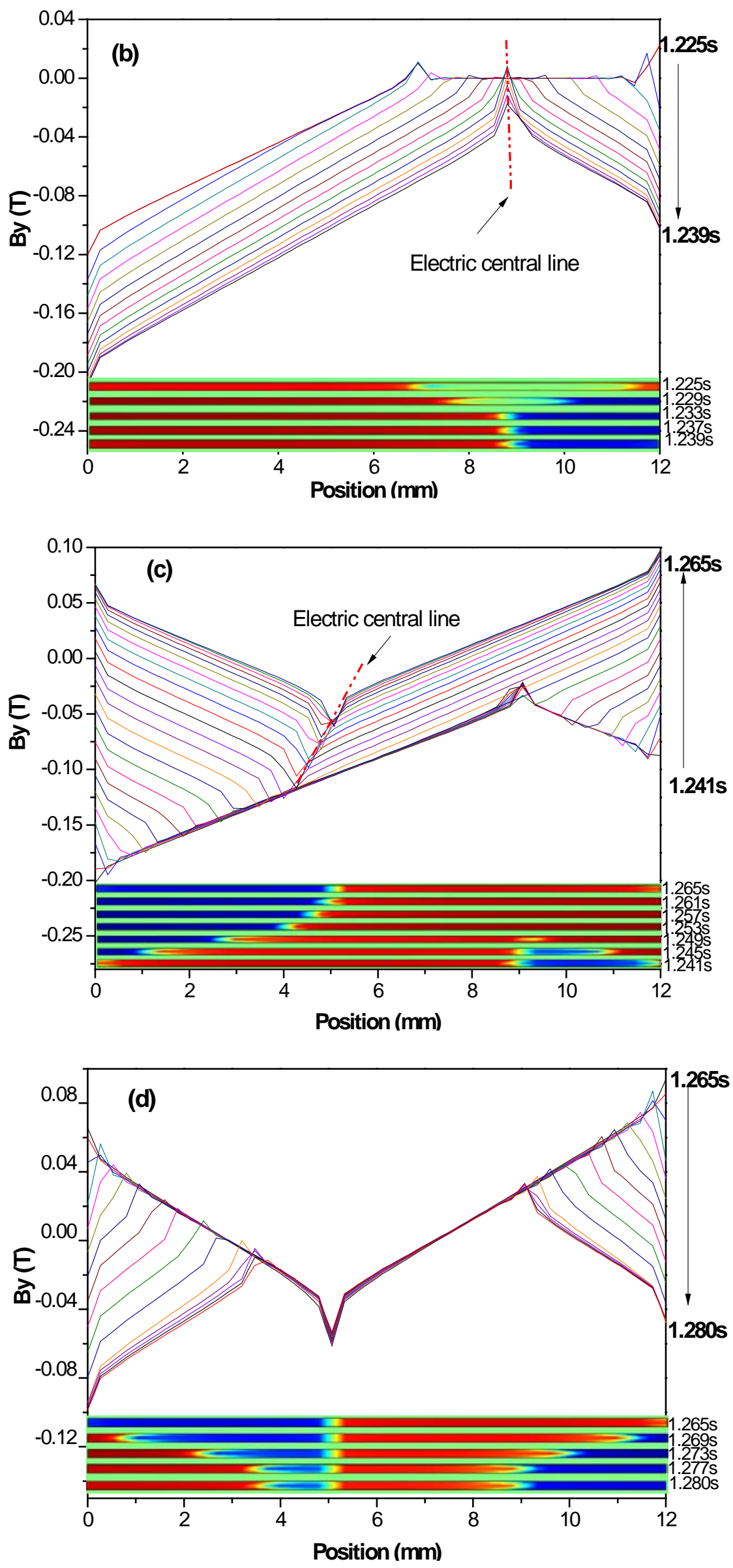
Fig. 10. Current and magnetic field distributions in the strip $S_{2}$ during the time when field is applied to it. The position zero indicates its left edge, and the position $12 \mathrm{~mm}$ indicates the right edge. The vertical magnetic flux density $B_{\mathrm{y}}$ is the value from the middle line of strip $S_{2}(y=0)$ across its width. The magnitude of $i_{\text {right }}$ is $500 \mathrm{~A}$.

A clear picture of how flux has travelled across strip $S_{2}$ is shown in Fig. 10. The position of interest is chosen to be the middle line of strip $S_{2}(y=0)$ across its width. The figure shows the flux density in $y\left(B_{y}\right)$ direction across the line, and the current distribution in it. The position zero indicates the left edge of $S_{2}$ and the position $12 \mathrm{~mm}$ indicates the right edge of $S_{2}$.

Fig. 10(a) shows the time period $1.220 \mathrm{~s}$ to $1.225 \mathrm{~s}$, when the global screening current induced by the central magnets reaches its positive peak, and before the field is applied to $S_{2}$. During this time period the current is relatively stable, and mostly distributed in the left side. There is virgin area in the strip where no current has penetrated in. The slope of the vertical magnetic flux density $\left(B_{\mathrm{y}}\right)$ is in accordance with the current distribution. In the virgin area, $B_{\mathrm{y}}$ is close to zero.

Fig. 10(b) shows the time period $1.225 \mathrm{~s}$ to $1.239 \mathrm{~s}$, when the field applied to $S_{2}$ changes from zero to negative maximum. The total flux in the strip (absolute value) increases. Flux enters the strip from both edge, but more are from the left than from the right because there is an initial "transport current". The screening current induced by the right magnet "pushes" the initial current at the left side further into the strip, whereas it changes the current direction at the left side. At about $1.233 \mathrm{~s}$, the strip is fully occupied by the initial "transport current" and the screening current. The positive current and negative current meet at the position near the right edge, which is referred as the electric central line. After that, the screening current does not increase much with the increase of applied field.

Fig. 10(c) shows the time period $1.241 \mathrm{~s}$ to $1.265 \mathrm{~s}$, when the field applied to $S_{2}$ increases from the negative maximum to the positive maximum. Before $1.249 \mathrm{~s}$, the screening current changes direction at both edge, and the slope of $B_{\mathrm{y}}$ also changes near the edges. But the in the center area both the current and $B_{\mathrm{y}}$ stay unchanged, seeming to be "frozen". The field change from $1.241 \mathrm{~s}$ to $1.249 \mathrm{~s}$ can be considered as the threshold value [30][31] in the dynamic resistance model, under which the applied field cannot interact with the transport current. At $1.249 \mathrm{~s}$ the $B_{\mathrm{y}}$ changes at all positions of the strip. The electric central line now has been shifted to the left. But its position varies with the increase of the applied field. This is because the "transport current" in the strip is not stable. It decreases when the field is applied to $S_{2}$, and also some current has bypassed by $S_{\mathrm{L}}$ resulting in an increase in $i_{\mathrm{L}}$.

Fig. 10(d) shows the time period $1.265 \mathrm{~s}$ to $1.280 \mathrm{~s}$, when the field applied to $S_{2}$ decreases from the positive maxima to zero. During which time only the current and field near the edges change direction.

Comparing Fig. 10(b) a Fig. 10(c), we can clearly see that the electric central line has been shifted by the "transport current" induced by the central magnet. Therefore, there is a net flux motion across the strip.

At the end of this Section, we show the current evolution in $S_{\mathrm{L}}$ during the first few cycles of charging in Fig. 11. 


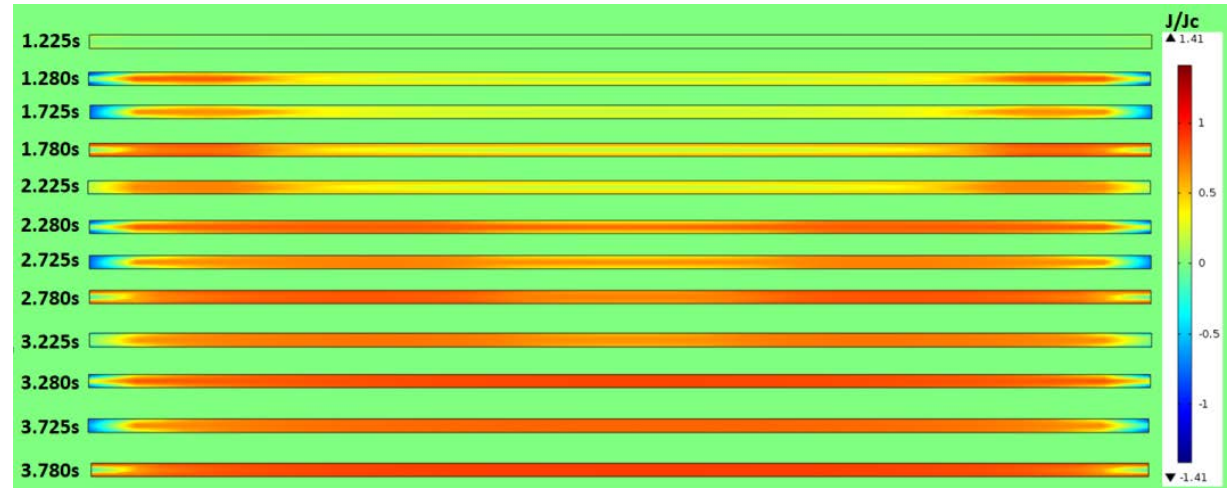

Fig. 11. Current evolution in the strip $S_{\mathrm{L}}$ during muli-cycles of applied field.

\subsection{Threshold magnetic field}

Another issue which is of importance is the threshold field [30][31], which is normally used to determine the minimum field to generate dynamic resistance. In the dynamic resistance model, transport current is assumed to flow in the central part of a strip, and rectifying effect only occurs if the applied field is large enough to penetrate to the area flowing transport current. Fig. 12 shows a case in which the applied field is slightly lower the threshold value. During the whole cycle of the switching field, there is an area in the center of the tape $(8-10 \mathrm{~mm})$ staying in "virgin state", without current or field. In this case, no flux tends to be able to travel across the strip. However, if we look at the current distribution in $S_{\mathrm{L}}$ during this period in Fig. 13, we can still see some weak pumping effect. This may be caused by the following factors. The first is that the strip is thin, so that some current tends to flow at the top and bottom surface of the strip rather than penetrates from the left and right edges to the center in a slab geometry. This may result in rectifying effect. The second is that the initial "transport current" is flowing near the edges rather than in the center and the applied field can interact with it, changing the current and field distribution, hence the mutual induction between the charging loop and the load loop. In this case the "pumped" current is more like a screening current rather than a really injected current. The third is that the threshold field previously studied is based on static analysis, assuming that the field changing rate is infinitely slow, which is not the actual case in our simulation.

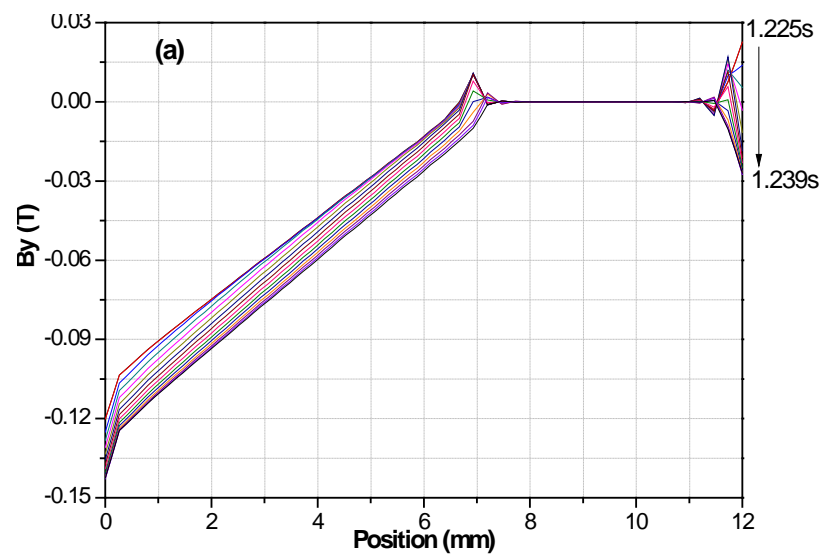



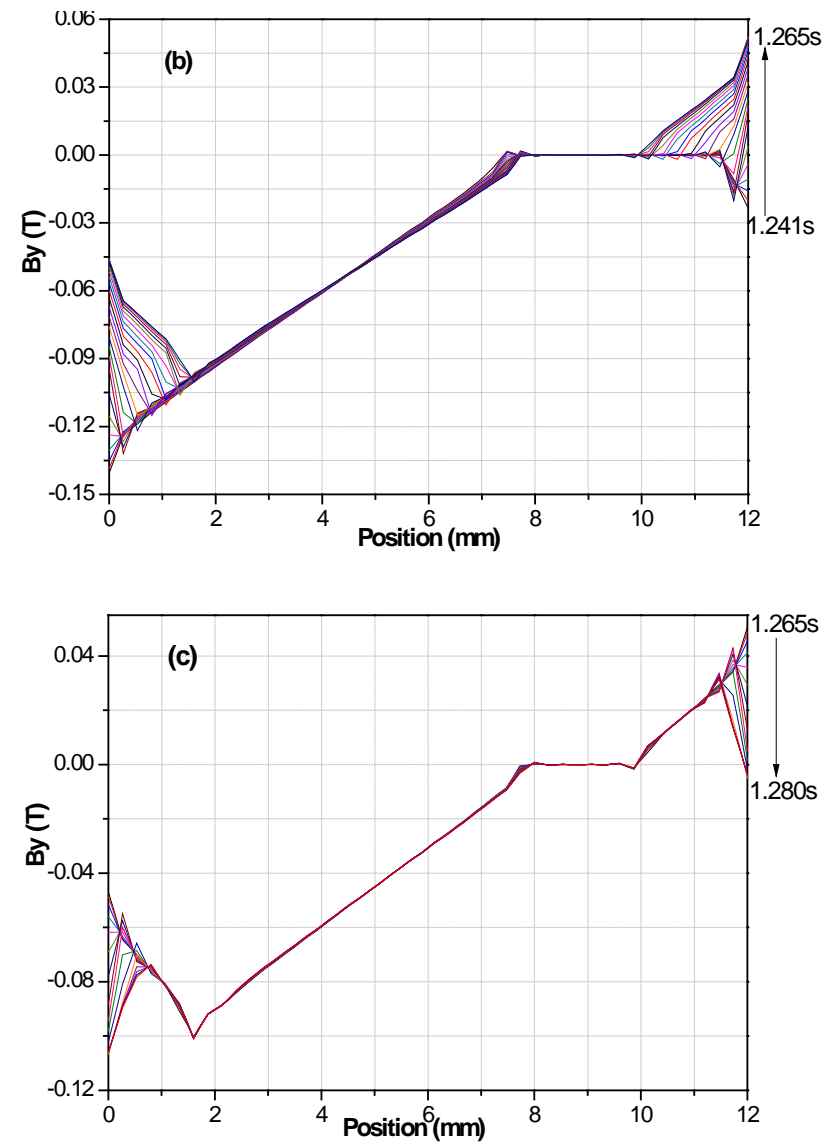

Fig. 12. Magnetic field distributions in the strip $S_{2}$ when the switching field magnitude is under the threshold value. The magnitude of $i_{\text {right }}$ is chosen to be $100 \mathrm{~A}$.

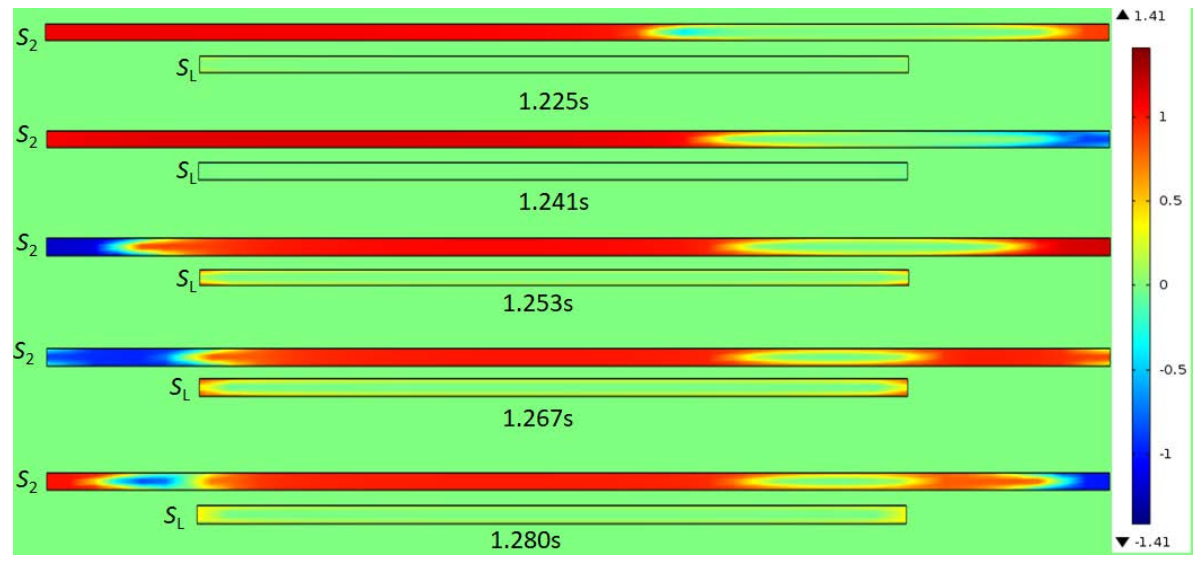

Fig. 13. Current distribution when the applied field is close to the threshold value.

\section{Discussion}

Fig. 10 clearly shows how flux can travel across an HTS strip. The central magnet induces a global screening current flowing around the charging loop formed by $S_{1}$ and $S_{2}$, and the left and right (the above result only shows the right) magnet induce a local screening current in an individual strip. If the strip is full of current, the local applied field interacts with the global 
screening current, resulting in a rectifying effect. This is very much similar to the well-known effect of dynamic resistance where a direct current carrying type II superconductor is under a perpendicular AC field. Although the induced global screening current is not a DC, its frequency in the simulation is much lower than the applied field frequency, so similar effect can be seen. Actually it is not necessary for the frequency of these two fields to be different from each other, and the rectifying effect occurs as long as the two fields are strong enough. Supposing that two magnetic fields are applied perpendicular to different parts of a thin strip, each of them tends to generate a screening current (inducing an electric field) in the superconductor. If these two magnetic fields are applied individually, the electric fields induced at a certain point of the superconductor may be different from each other. And if the fields are applied together, the electric field at the point cannot have two different values at the same time, which means there is rectifying effect in the superconductor. In this case, it may difficult to tell which is the magnetic field inducing the global "transport current", and which is the switching field.

Over and above all, for flux pumping to work under perpendicular field, the prerequisite is that the superconductor has to be thin. If the thickness is much larger than the width of the superconductor (slab geometry), or the thickness is much larger than the radius of the loop formed by the superconductor (thick superconducting rings), flux pumping is less likely to happen. Because the inhomogeneous applied field will become homogeneous in the middle part of a superconductor due to screening effect in the top and bottom surfaces. Thus the field distribution will follow the Bean's Model in the middle.

\section{Conclusion}

In this work, we proposed a finite element modeling method to simulate dynamic resistance switched transformer-rectifier flux pump using 2D H-formulation. The simulation result is in well accordance with our experimental data. The result has clearly shown how flux has traveled across an HTS strip without driving the superconductor normal. The model also shows that it is not necessary for the superconductor to be $J_{\mathrm{c}}(B)$ dependent to achieve flux pumping, and indicates that the behavior may also occur in hard superconductors.

\section{References}

[1] I. Giaever, IEEE Spectrum 3, 117 (1966).

[2] L. J. M. van de Klundert and H. H. J. ten Kate, Cryogenics 21, 195 (1981).

[3] L. J. M. van de Klundert and H. H. J. ten Kate, Cryogenics 21, 267 (1981).

[4] Z. Bai, G. Yan, C. Wu, S. Ding, and C. Chen, Cryogenics 50(10), 688(2010).

[5] C. Hoffmann, D. Pooke, and A. D. Caplin, IEEE Trans. Appl. Supercond.21, 1628 (2011).

[6] Z. Jiang, K. Hamilton, N. Amemiya, R. A. Badcock, and C. W. Bumby, Appl. Phys. Lett. 105, 112601 (2014).

[7] J. Geng and T. A. Coombs, Appl. Phys. Lett. 107, 142601 (2015).

[8] J. Geng and T. A. Coombs, Supercond. Sci. Technol. 29, 095004 (2016).

[9] E. Pardo, Supercond. Sci. Technol. 30, 060501 (2017).

[10] J. Geng et al, J. Phys. D: Appl. Phys. 49, 11LT01 (2016).

[11] V. V. Andrianov, V. B. Zenkevich, V. V. Kurguzov, V. V. Sychev, and F.F. Ternovskii, Sov. Phys. JETP 31, 815 (1970).

[12] R. P. Huebener, L. G. Stafford, and F. E. Aspen, Phys. Rev. B 5, 3581(1972).

[13] T. Ogasawara, K. Yasukochi, S. Nose, and H. Sekizawa, Cryogenics 16(1), 33 (1976) 
[14] M. P. Risse, M. G. Aikele, S. G. Doettinger, and R. P. Huebener, Phys.Rev. B 55, 15191 (1997).

[15] J. J. Rabbers, B. ten Haken, F. Gomory, and H. H. J. ten Kate, Physica C 300, 1 (1998).

[16] M. P. Oomen, J. Rieger, M. Leghissa, B. ten Haken, and H. H. J. ten Kate, Supercond. Sci. Technol. 12, 382 (1999).

[17] G. P. Mikitik and E. H. Brandt, Phys. Rev. B 64, 092502 (2001).

[18] A. Uksusman, Y. Wolfus, A. Friedman, A. Shaulov, and Y. Yeshurun, J. Appl. Phys. 105(9), 093921 (2009).

[19] Y. Yoshida, M. Uesaka, and K. Miya, IEEE Trans. Magn., 30, 3503(1994).

[20] A. Gladun, G. Fuchs, K. Fischer, D. Busch, R. Eujen, and J. Huedepohl, IEEE Trans. Appl. Supercond. 3, 1390 (1993).

[21] C. P. Bean, “Magnetization of high-field superconductors,” Rev. Mod. Phys., 36, 31(1964).

[22] A. M. Campbell, Supercond. Sci. Technol. 30, 125015 (2017).

[23] Q. Li, M. Yao, Z. Jiang, C. W. Bumby, N. Amemiya, IEEE Trans. Appl. Supercond.28, 6600106 (2018).

[24] M. D. Ainslie, C. W. Bumby, Z. Jiang, R. Toyomoto, N. Amemiya, Supercond. Sci. Technol. 31, 074003 (2018).

[25] Z. Hong, A. M. Campbell and T. A. Coombs, Supercond. Sci. Technol. 19, 1246-1252(2006).

[26] Ph. Vanderbemden et al, Physical Review B 75, 174515 (2007).

[27] M. D. Ainslie et al, Supercond. Sci. Technol. 24, 045005 (2011).

[28] M. Zhang, J. Kim, S. Pamidi, M. Chudy, W. Yuan, TA Coombs, J. Appl. Phys. 111, 083902 (2012).

[29] J. Geng et al, Supercond. Sci. Technol. 29, 035015(2016).

[30] Z. Jiang, R. Toyomoto, N. Amemiya, X. Zhang, and C. W. Bumby, Supercond. Sci. Technol. 30, 03LT01(2017).

[31] Z. Jiang et al, IEEE Trans. Appl. Supercond.28, 8200305 (2018). 